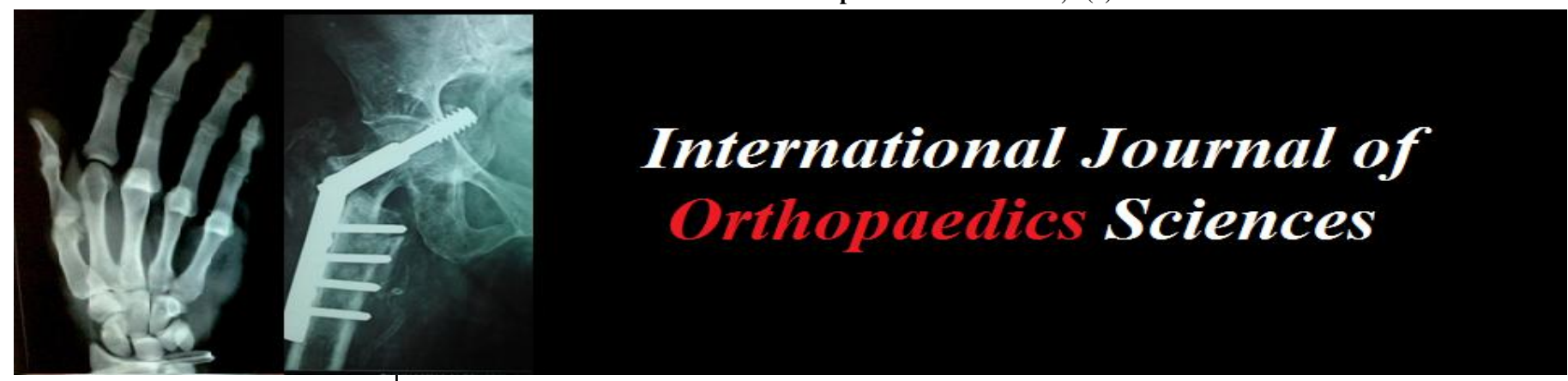

ISSN: $2395-1958$

IJOS 2018; 4(1): 667-669

(C) 2018 IJOS

www.orthopaper.com

Received: 17-11-2017

Accepted: 18-12-2017

Nicholas Kolodychuk

University of Queensland School of Medicine, Australia
1

Correspondence

Nicholas Kolodychuk

University of Queensland School

of Medicine, Australia

\section{Tendinosis as the underlying pathology of osgood- schlatter disease: imaging similarities and treatment implications}

\section{Nicholas Kolodychuk}

DOI: https://doi.org/10.22271/ortho.2018.v4.i1j.97

\section{Abstract}

Osgood-Schlatter disease is relatively common condition in adolescents. Classically, its pathophysiology is considered to be a traction apophysitis of the proximal tibial tubercle, which is caused from overuse. However, there is growing evidence that the underlying pathology of Osgood-Schlatter disease is tendinosis rather than apophysitis. Tendinosis is described as a degeneration of collagen following chronic overuse of a tendon. Examination of ultrasonographic and magnetic resonance imaging of tendinosis exhibits many similarities with the imaging of Osgood-Schlatter disease. Further research should be conducted in implicating tendinosis as the pathologic mechanism in Osgood-Schlatter disease. This paper provides a brief overview explaining the basis of tendinosis as the basic pathophysiology underlying Osgood-Schlatter disease and explores the related treatment implications.

Keywords: Osgood-Schlatter disease; tendinosis; pathology; imaging; knee pain; treatment

\section{Introduction}

Osgood-Schlatter disease is an overuse injury of the anterior knee occurring in adolescents originally described by Robert Osgood [1] and Carl Schlatter ${ }^{[2]}$ in 1903. It presents with localized pain to the insertion of the patellar ligament at the tibial tuberosity. The current consensus on the pathophysiology of OSD is that it is a traction apophysitis of the proximal tibial tubercle resulting from repetitive microtrauma ${ }^{[3,4]}$. Tendinopathy is defined as clinical presentation of pain resulting in loss of function with possible swelling and thickening ${ }^{[5]}$. The pathological signs known as tendinosis include a myriad of histopathologic changes: hypervascularity, increased innervation, and hypercellularity ${ }^{[6]}$. Use of ultrasonographic imaging has been shown to provide reliable and reproducible results in imaging in of the patellar tendon ${ }^{[7]}$. Similar pathologic findings have been noted in studies of OSD ${ }^{[8,9]}$. These similarities between OSD with the pathophysiology known as tendinosis suggest new treatment protocols, new aetiology, and possible genetic links for OSD. The basis of OSD pathology in tendinosis will be discussed further in this paper.

\section{Materials and methods}

The authors searched PubMed and EMBASE using the search terms, "Osgood Schlatter disease" and " "patellar tendinosis athletes' OR 'patellar tendinosis adolescents"”. Results were limited to articles from 2009-2014, returning 209 results. Duplicates were removed leaving 173 articles. Titles and abstracts were scanned for relevance to imaging, non-operative treatment, and pathology resulting in 55 articles being examined thoroughly. References were canvassed for further articles.

\section{Results and Discussion}

Tendinosis is the basic pathophysiology that underlies many tendon disorders. It is described as a degeneration of collagen in a tendon caused by chronic overuse ${ }^{[10]}$. As overuse occurs without sufficient time for healing the collagen of the tendon degenerates and its organization becomes disorganized. These changes can be seen histologically and in both magnetic resonance imaging and ultrasonographic imaging ${ }^{[11]}$. Histopathological analysis of tendons 
experiencing tendinosis have been noted to have downregulation of Type I collagen and fibromodulin and an upregulation of Type III collagen and versican ${ }^{[12]}$. This ratio between Type 1 and Type 3 collagen relate to the strength of the tendon, as Type 1 collagen is stronger. The increased Type 3 collagen is disorganized and the fibrils no longer align with each other, there may also be decreased linking between fibrils ${ }^{[10,12]}$. Biochemical studies have identified plateletderived growth factor receptor $\beta$ (PDGFR $\beta$ ) expression to be related to hypercellularity in patellar tendinosis ${ }^{[13]}$. Increased expression of vesicular glutamate transporters (VGluT2) has been noted in tenocytes in pathologic patellar tendons, which may be important due to glutamate's role in controlling apoptosis, proliferation, and necrosis ${ }^{[14]}$.

MRI of tendons with tendinosis shows the following signs: hyperintensity and increased thickness of the tendon ${ }^{[11]}$. Both of these changes are commonly seen in tendinosis of the patellar tendon, which is the tendon affected in OSD ${ }^{[15]}$. US imaging can be used to visualize the pathologic changes to tendons in tendinosis. Commonly US imaging shows decreased echogenicity, increased tendon thickness, calcium deposits, and neovascularization when using Doppler ${ }^{[11]}$.

OSD is an overuse injury in adolescents to the patellar tendon at its insertion to the tibial tuberosity. It commonly presents with anterior knee pain, which can be localized to the tibial tuberosity. The changes in PDGFR $\beta$ and VGluT2 expression that have been described in patellar tendinosis should also be investigated to determine any role they may have in the pathophysiology of OSD. Although diagnosis of OSD is primarily clinical, MRI and US imaging can be used to demonstrate pathologic changes and confirm diagnosis.

Common MRI findings in OSD show hypertrophy or fragmentation of the tibial tubercle with possible presence of ossification within the distal patellar tendon ${ }^{[16]}$. Edema adjacent to the patellar tendon often occurs ${ }^{[17]}$. Additionally thickening of the patellar tendon, loss of the sharp inferior angle of the infrapatellar fat pad and surrounding soft tissue, and/or infrapatellar bursitis may be noted [17-19]. Use of US imaging has been investigated by Czyrny and Greenspan [20${ }^{22}$. The most notable pathologic features seen in US imaging of OSD are: presence of a hypoechoic zone superficial to the apopohysis of the anterior tibial tubercle, a fragmented and hypoechoic ossification center, diffuse thickening of the insertion portion of the patellar tendon, and patellar bursitis (fluid collection in the soft tissues deep to the patellar tendon). Hypervascularity adjacent to the attachment of the patellar tendon has also been noted on US with Doppler [17, 20, 23, 24].

The pathologic signs seen in MRI and US imaging, as just discussed, have significant overlap between the classic signs of tendinosis and OSD. Due to lack in consensus of the aetiology of OSD investigation of these similar findings is warranted. The similarities between the basic pathophysiology of tendinosis and OSD may also prove useful in examining treatment options for OSD, as these findings suggest OSD is at least partly the result of tendinosis. There are numerous treatment options currently in use for tendinosis. One of the primary treatments is the use of NSAIDs, ice, and rest ${ }^{[12]}$. This is commonly used as a firstline approach before more invasive treatments are considered. Several types of injection therapies can be used in treatment of tendinosis: dry needling, autologous blood, platelet-rich plasma, prolotherapy, and high volume injections ${ }^{[12]}$. The general mechanisms of these treatments can be broken into two groups: (a) those increase healing of the tendon and (b) those that target neovascularization. Dry needling, autologous blood, and platelet rich plasma aim to increase healing of the tendon by activating inflammatory cascades ${ }^{[25]}$. Prolotherapy and high volume injections target the neovascularization that is often present in tendinosis ${ }^{[12,25]}$. To accomplish this US should be used to guide a needle into the region of the neovascularization then the sclerosing agent should be injected. There are numerous sclerosing agents (dextrose, polidocanol, phenol-gylcerine-glucose, and sodium morrhuate) that can be used ${ }^{[25]}$. The result is a decrease in neovascularization in the area surrounding the tendon, which has been correlated to decreased pain in the tendon ${ }^{[9,12,24]}$. High volume injections aim to accomplish the same result by injecting high volumes of fluid which interferes with the neovascularization process ${ }^{[12]}$.

Platelet-rich plasma has been shown to be effective in treating tendinosis $[25,29,30]$. The PRP contains high concentrations of numerous growth factors such as: insulin-like growth factor (IGF-1), transforming growth factor (TGF- $\beta$ ), platelet-derived growth factor (PDGF), vascular endothelial growth factor (VEGF), fibroblast growth factor (FGF), platelet-derived angiogenic factor (PDAF), and platelet-derived endothelial growth factor (PDEGF) ${ }^{[30]}$. The injection leads to increased extracellular matrix synthesis, cell migration, and proliferation resulting in healing to the injured area of tendon ${ }^{[29]}$. The increase in these processes aids in the reorganization and strengthening of the pathologic tendon in tendinosis. As previously discussed tendinosis is indicated by changes in collagen type and correct organization of the collagen. PRP increases healing through stimulation of fibroblasts and other cells in the area.

Current treatment protocol for OSD is conservative, consisting primarily of rest, ice, NSAIDs, and possibly physical therapy ${ }^{[26]}$. Conservative treatment is successful in approximately $90 \%$ of cases, although it may take 2 years for resolution of symptoms and return to normal activity levels. Topol et al. ${ }^{[9]}$ described positive results for treating OSD with injections of $1 \%$ lidocaine/12.5\% dextrose solution in their randomized control trial. This treatment was investigated following evidence of its efficacy in treating tendinosis [27, 28]. The promising results of lidocaine+dextrose prolotherapy in treating OSD provide further evidence that the pathologic process at the root of OSD is tendinosis. Thusly, other treatment options currently in use for tendinosis should be investigated for their use in treating OSD. High volume injections and the use of additional sclerosing agents seem particularly warranted following the success of dextrose prolotherapy due to the similar mechanism in decreasing neovascularization. Currently there is a paucity of research into the use of tendon healing treatments that are effective in tendinosis for OSD. Clinical trials on the use of autologous blood injections or PRP injections in treatment of OSD are warranted.

\section{Conclusion}

Osgood-Schlatter disease exhibits numerous hallmark signs of tendinosis as its underlying basic pathology. In OSD tendon thickness is increased, the tendon appears with decreased intensity upon US imaging, and US with Doppler shows neovascularization surrounding the injured tendon, which have all been described as signs of tendinosis. There has been significant research into various treatment options for tendinosis, however few of these have been tried in treatment of OSD. Lidocaine+dextrose prolotherapy treatment has been shown to be effective in treating tendinosis, and has also been 
effective in OSD treatment. It seems likely that tendinosis is a large part of the underlying pathology of OSD, meaning that OSD will respond to treatments used for tendinosis. Thorough investigation of treatments that are currently used in tendinosis for their efficacy in treating OSD is required before they can be widely recommended to practioners. These investigations may lead to breakthroughs in OSD treatment, leading to new therapeutic options for when traditional conservative treatment proves ineffective.

\section{References}

1. Osgood RB. Lesions of the tibial tubercle occurring during adolescence. Clin Orthop. 1903; (286):4-9.

2. Schlatter C. Verletzungen des schnabelformigen: Fortsatzes der oberen tibiaepiphyse. Beitr Klin Chir. 1903; (38):874.

3. Ogden JA, Southwick WO. Osgood-Schlatter's disease and tibial tuberosity development. Clin Orthop. 1976; (116):180-9.

4. Ogden JA. Radiology of postnatal skeletal development. X. Patella and tibial tuberosity. Skeletal Radiol. 1984; 11(4):246-57.

5. Cassel M, Baur H, Hirschmüller A, Carlsohn A, Fröhlich K, Mayer F. Prevalence of Achilles and patellar tendinopathy and their association to intratendinous changes in adolescent athletes: Tendinopathy in adolescent athletes. Scand J Med Sci Sports. 2014 Oct; $/ \mathrm{a}-\mathrm{n} / \mathrm{a}$.

6. Fu S-C, Rolf C, Cheuk Y-C, Lui PP, Chan K-M. Deciphering the pathogenesis of tendinopathy: a threestages process. BMC Sports Sci Med Rehabil. 2010; 2(1):30.

7. Sunding K, Fahlström M, Werner S, Forssblad M, Willberg L. Evaluation of Achilles and patellar tendinopathy with greyscale ultrasound and colour Doppler: using a four-grade scale. Knee Surg Sports Traumatol Arthrosc [Internet]. 2014 Sep 6 [cited 2014 Nov 26]; Available from: http://link.springer.com/10.1007/s00167-014-3270-4

8. Kaya DO, Toprak U, Baltaci G, Yosmaoglu B, Ozer H. Long-term functional and sonographic outcomes in Osgood-Schlatter disease. Knee Surg Sports Traumatol Arthrosc. 2013; 21(5):1131-9.

9. Topol GA, Podesta LA, Reeves KD, Raya MF, Fullerton BD, Yeh H-w. Hyperosmolar Dextrose Injection for Recalcitrant Osgood-Schlatter Disease. PEDIATRICS. 2011; 128(5):e1121-8.

10. Bass E. Tendinopathy: Why the difference between tendinitis and tendinosis matters. Int $\mathbf{J}$ Ther Massage Bodyw. 2012; 5(1):14.

11. Weinreb JH, Sheth C, Apostolakos J, McCarthy M-B, Barden B, Cote MP, et al. Tendon structure, disease, and imaging. Muscle Ligaments Tendons J. 2014; 4(1):66-73.

12. Christian RA, Rossy WH, Sherman OH. Patellar Tendinopathy. Bull Hosp Joint Dis. 2014; 72(3).

13. Rolf CG, Fu BS, Pau A, Wang W, Chan B. Increased cell proliferation and associated expression of PDGFRbeta causing hypercellularity in patellar tendinosis. Rheumatol Oxf Engl. 2001; 40(3):256-61.

14. Scott A, Alfredson H, Forsgren S. VGluT2 expression in painful Achilles and patellar tendinosis: Evidence of local glutamate release by tenocytes. J Orthop Res. 2008; 26(5):685-92.

15. Pearson SJ, Hussain SR. Region-Specific Tendon Properties and Patellar Tendinopathy: A Wider
Understanding. Sports Med. 2014; 44(8):1101-12.

16. Sanchez R, Strouse PJ. The Knee: MR Imaging of Uniquely Pediatric Disorders. Magn Reson Imaging Clin N Am. 2009; 17(3):521-37.

17. Morrow MS, Oliveira AM. Imaging of Lumps and Bumps in Pediatric Patients: An Algorithm for Appropriate Imaging and Pictorial Review. Semin Ultrasound CT MRI. 2014; 35(4):415-29.

18. Chang GH, Paz DA, Dwek JR, Chung CB. Lower extremity overuse injuries in pediatric athletes: clinical presentation, imaging findings, and treatment. Clin Imaging. 2013; 37(5):836-46.

19. Rosenberg ZS, Kawelblum M, Cheung YY, Beltran J, Lehman WB, Grant AD. Osgood-Schlatter lesion: fracture or tendinitis? Scintigraphic, CT, and MR imaging features. Radiology. 1992; 185(3):853-8.

20. Czyrny Z. Osgood-Schlatter disease in ultrasound diagnostics-a pictorial essay. Med Ultrason. 2010; 12(4):323-35.

21. Czyrny Z, Greenspan A. Osgood-Schlatter Disease: Effectiveness of US Imaging. Osteol KÖZlemények. 2011; (2):59-66.

22. Czyrny Z, Greenspan A. Osgood-Schlatter disease: a new perspective and classification based on ultrasonography. Ultrasonographia. 2009; (38):55-70.

23. Davis KW. Imaging Pediatric Sports Injuries: Lower Extremity. Radiol Clin North Am. 2010; 48(6):1213-35.

24. Sailly M, Whiteley R, Johnson A. Doppler ultrasound and tibial tuberosity maturation status predicts pain in adolescent male athletes with Osgood-Schlatter's disease: a case series with comparison group and clinical interpretation. Br J Sports Med. 2013; 47(2):93-7.

25. Judson $\mathrm{CH}$, Wolf JM. Lateral Epicondylitis. Orthop Clin North Am. 2013; 44(4):615-23.

26. Gholve PA, Scher DM, Khakharia S, Widmann RF, Green DW. Osgood Schlatter syndrome. Curr Opin Pediatr. 2007; 19(1):44-50.

27. Ryan M, Wong A, Taunton J. Favorable Outcomes After Sonographically Guided Intratendinous Injection of Hyperosmolar Dextrose for Chronic Insertional and Midportion Achilles Tendinosis. Am J Roentgenol. 2010; 194(4):1047-53.

28. Yelland MJ, Sweeting KR, Lyftogt JA, Ng SK, Scuffham PA, Evans KA. Prolotherapy injections and eccentric loading exercises for painful Achilles tendinosis: a randomised trial. Br J Sports Med. 2011; 45(5):421-8.

29. Di Matteo B, Filardo G, Kon E, Marcacci M. Platelet-rich plasma: evidence for the treatment of patellar and Achilles tendinopathy-a systematic review. Musculoskelet Surg [Internet]. 2014 Oct 17 [cited 2014 Nov 26]; Available from: http://link.springer.com/10.1007/s12306-014-0340-1

30. Jeong DU, Lee C-R, Lee JH, Pak J, Kang L-W, Jeong $\mathrm{BC}$, et al. Clinical Applications of Platelet-Rich Plasma in Patellar Tendinopathy. BioMed Res Int. 2014; 2014:115. 